\section{RIEGO DEFICITARIO EN MELOCOTONERO. ANÁLISIS DEL RENDIMIENTO Y DE LA EFICIENCIA EN EL USO DEL AGUA}

\section{PEACH DEFICIT IRRIGATION. FRUIT YIELD AND WATER USE EFFICIENCY ANALYSIS}

\author{
Mario Tapia Vargas ${ }^{1 *}$, Antonio Larios Guzmán ${ }^{1}$, \\ Isabel Abrisqueta Villena ${ }^{2}$, Oussama Mounzer ${ }^{2}$, \\ Juan Vera Muñoz ${ }^{2}$, José M. Abrisqueta $\operatorname{García}^{2}$ y \\ M. Carmen Ruiz Sánchez ${ }^{2}$
}

${ }^{1}$ Campo Experimental Uruapan, Instituto Nacional de Investigaciones Forestales, Agrícolas y Pecuarias. 60080, Av. Latinoamericana 1101. Uruapan, Mich. Tel. 452-523-7392 Ext. 101. ${ }^{2}$ CEBAS-CSIC. Campus Universitario Espinardo. 30100, Murcia, España.

* Autor para correspondencia (tapia.luismario@inifap.gob.mx)

\section{RESUMEN}

El objetivo de este trabajo fue estudiar el efecto de distintas estrategias de riego deficitario en las relaciones hídricas, el rendimiento y calidad del fruto y la eficiencia del uso del agua en melocotón (Prunus persica L. Batsch). Este ensayo se estableció durante el año 2007 en una finca experimental ubicada, en Santomera, Murcia, España con la variedad 'Flordastar', en riego por goteo. Los tratamientos evaluados fueron: T1, testigo con riego diario equivalente a $120 \%$ de la evapotranspiración de cultivo (ETc); T2, con riego restringido todo el ciclo (50 \% de ETc); T3, con riego deficitario en el periodo de postcosecha (resto normal); y T4, con umbral de riego automatizado a un consumo máximo de $10 \%$ de la capacidad de campo. El diseño experimental fue en bloques al azar con cuatro repeticiones por tratamiento (dentro de una hilera de 13 árboles por repetición). Se determinó la eficiencia de uso de agua ( $\mathrm{kg}$ de fruto $\mathrm{m}^{-3}$ de agua ET) mediante la relación rendimiento de fruto y la evapotranspiración real (ETr). Los resultados indicaron que T1 tuvo menor $(P \leq 0.05)$ eficiencia en el uso de agua con 2.1, que $\mathrm{T} 4$ con 2.8. Los tratamientos $\mathrm{T} 2$ y $\mathrm{T} 3$ superaron $(\mathrm{P} \leq \mathbf{0 . 0 5})$, a $\mathrm{T} 1$ con valores de 2.7 y 3.0, lo que muestra que el árbol se ajusta a los cambios en el manejo del riego, para mantener o incluso mejorar, la eficiencia en el uso del agua.

Palabras clave: Prunus pérsica, balance hídrico, riego localizado.

\section{SUMMARY}

The purpose of this work was to study the effect of different deficit irrigation strategies on water relations, fruit quality, yield, and water use efficiency of peach trees (Prunus persica L.). This assay was established during 2007 at an experimental station in Santomera, Murcia, Spain, with var. 'Flordastar', growing under drip irrigation. The evaluated treatments were: T1, control with dai- ly irrigation equivalent to $120 \%$ of the crop evapotranspiration (ETc); T2, restricted irrigation on the whole cycle (50\% of ETc); T3, restricted irrigation during the post-harvest period (rest of the cycle normal irrigation); and T4, an automated threshold irrigation at a maximum of $10 \%$ of field capacity. Experimental design was completed randomized with four replications (within a row of 13 trees per replication). Water use efficiency (kg of fruit per $\mathrm{m}^{-3}$ of ET water) was determined by relating fruit yield and real evapotranspiration (ETr). Results showed that $\mathrm{T} 1$ had the lowest $(\mathrm{P} \leq \mathbf{0 . 0 5})$ water use efficiency with 2.1 , compared to $\mathrm{T} 4$ with 2.8 . Treatments T2 and T3 were also higher $(P \leq \mathbf{0 . 0 5})$ than $T 1$, with values of 2.7 and 3.0, respectively. These results show trees are able to adjust to changes in water management, keeping and even improving water use efficiency.

Index words: Prunus persica, water balance, peach, drip irrigation.

\section{INTRODUCCIÓN}

La principal característica del agro en el mediterráneo español es la escasez de agua y la intensa competencia que existe por su uso con otros sectores de la economía como servicios, industria y urbano. La agricultura utiliza más de $70 \%$ del agua dulce disponible por lo que la necesidad de incrementar la eficiencia del uso del agua ha propiciado la búsqueda de mejores tecnologías en el riego (Abrisqueta et al., 2008). La región de Murcia se distingue por tener el mayor impulso de esquemas de riego de bajo consumo de agua, con $76 \%$ de 115000 ha de riego cultivadas bajo riego de baja presión, que convierte a esta comunidad como la que mejor cuida el agua a nivel europeo (Mounzer et al., 2008a). Esto ha ocurrido, entre otras razones, por el alto precio del agua $\left(0.40 € \mathrm{~m}^{-3}\right)$ para los productores murcianos (Ballesteros, 2005). Por ello, la agricultura española actual se enfoca a maximizar la productividad del agua (Playan y Mateos, 2006).

La horticultura de Murcia es conocida como el surtidor de frutas y hortalizas de alta calidad al resto de Europa, por lo que este sector representa $13 \%$ de la economía regional y es altamente generador de empleos. Sin embargo, el hecho de tener más de $1000 \mathrm{~mm}$ anuales de evapotranspiración potencial (ETo), que representa una alta demanda por el recurso hídrico, sobre todo en cultivos perennes como los frutales que presentan alto requerimiento de agua en el periodo de formación de fruto para no reducir el rendimiento, y durante el periodo de postcosecha para no afectar la producción del siguiente año (Torrecillas et al., 2000).

Debido a esta circunstancia, se han conducido diversas estrategias para generar información que permita hacer un uso más eficiente del agua, tales como reducción del riego de manera parcial o total (Martínez et al., 2007), uso de autómatas para el riego a determinados niveles de humedad (Mounzer et al., 2008a), optimización del riego 
con la utilización de sensores electrónicos del flujo de savia y de las variaciones micrométricas del tronco (Ortuño et al., 2006), y el estudio de la fisiología y el estado energético del agua en la planta (Conejero et al., 2007). En estas investigaciones se ha encontrado ahorros en la aplicación del agua de más de $500 \mathrm{~m}^{3} \mathrm{ha}^{-1} \mathrm{año}^{-1}$, sin afectar variables fisiológicas y energéticas. No obstante, aún falta evaluar el efecto del manejo del agua de riego en relación con la evapotranspiración real o actual (ETr) del cultivo, así como el efecto de la reducción del riego en la calidad o tamaño de fruto, a modo de dictaminar si el ahorro de agua se traduce en una mayor eficiencia del recurso o bien, si este ahorro afecta la calidad de fruto cosechado. El objetivo de este trabajo fue estudiar el efecto de varias estrategias de riego deficitario en las relaciones hídricas, el rendimiento y calidad del fruto, y la eficiencia del uso del agua en función del agua evapotranspirada.

\section{MATERIALES Y MÉTODOS}

Los ensayos se efectuaron en una parcela de 0.8 ha, de la finca experimental del Centro de Edafología y Biología Aplicada del Segura, en Santomera, Murcia, España ( $38^{\circ}$ 06' 31' LN, $1^{\circ}$ 02' 14' LO), con árboles de melocotón del cv. 'Flordastar', injertados sobre el patrón 'GF-677' de seis años de edad, a un marco de $5 \times 5 \mathrm{~m}$, durante el ciclo 2007. La parcela experimental fue dividida en cuatro subparcelas de 78 árboles cada una (6 filas x 13 árboles). El suelo es pedregoso, poco profundo y con una textura franco arcillosa (25\% de arena, $45 \%$ de limo y $30 \%$ de arcilla) y una densidad aparente media en un perfil de 80 $\mathrm{cm}$ de $1.42 \mathrm{~g} \mathrm{~cm}^{-3}$, clasificado como Lithic Xeric Haploxeroll (Soil Survey Staff, 1998).

La finca experimental dispone de una estación agrometeorológica constituida por sensores de temperatura, humedad relativa, radiación solar, velocidad del viento y lluvia, con sistema telemático de almacenamiento y servidor "web" via internet. El agua de riego procede del trasvase Tajo-Segura. El riego se realiza mediante un sistema independiente, que consiste en un único lateral por fila de árboles y ocho emisores autocompensantes por árbol, con un caudal nominal de $2 \mathrm{~L} \mathrm{~h}^{-1}$, situados a $0.5,1$, 1.5 , y $2 \mathrm{~m}$ respectivamente, a cada lado del tronco.

Se establecieron cuatro tratamientos de riego distribuidos al azar en cada subparcela (cuatro repeticiones) en función del criterio utilizado para su programación: T1, tratamiento testigo con programación del riego tradicional basada en la ecuación de PenmanMonteith (Allen et al., 1998), según los datos climáticos registrados por la estación agrometeorológica (ETo); la evapotranspiración del cultivo se repone en su totalidad más $20 \%(\mathrm{ETc}=\mathrm{ETo} \times \mathrm{Kc})$, para asegurar condiciones no limitantes de agua (120\% de la ETc). T2, básicamente es regado a $50 \%$ de la ETc. T3 es un tratamiento con riego restringido en postcosecha y riego con base en los datos de ETo, en el resto del ciclo vegetativo de septiembre a mayo. T4 es un tratamiento basado en umbrales de humedad, con riego automatizado que inicia al reducirse $10 \%$ la capacidad de campo del suelo (CC) y se suspende al alcanzar CC; en este tratamiento se utilizan sensores FDR ${ }^{\circledR}$ (Frequency Domain Reflectrometry, Agrilink Inc. Ltd., Adelaide, Australia) para normar el criterio de riego (Mounzer et al., 2008b).

El estudio de las relaciones hídricas incluyó el cálculo de la evapotranspiración real de cada tratamiento en las cuatro repeticiones, mediante la ecuación de conservación de la masa en el perfil 0-60 cm de suelo, cuya expresión es:

$$
E T r=I+R-D \pm \Delta S
$$

Donde: $I$ es el riego aplicado $(\mathrm{mm}) ; R$ es la lluvia registrada $(\mathrm{mm}) ; D$ es el drenaje o agua percolada mayor a $60 \mathrm{~cm}$ de profundidad $(\mathrm{mm}):$ y $\Delta S$ es el cambio en el almacenamiento de humedad de suelo $(\mathrm{mm})$.

El drenaje fue evaluado como el agua percolada en la profundidad $>60 \mathrm{~cm}$ del suelo. El balance se efectuó omitiendo el flujo lateral y capilar y considerando sólo el flujo vertical del suelo (Arregui y Quemada, 2006). La medición de la humedad en el suelo se hizo mediante tubos de acceso (un tubo por cada tratamiento y repetición), colocados uno en cada tratamiento y en las cuatro repticiones, para una sonda de neutrones instalada en una fuente radiactiva Troxler $3300 \AA$ (Troxler Electronic Laboratorios Inc., Research Triangle Park, NC, USA), como se ilustra en la Figura 1. Cada muestreo, constó de siete profundidades cada $10 \mathrm{~cm}$ desde 20 hasta $80 \mathrm{~cm}$. Para medir la humedad en la profundidad 0-10 cm se utilizó un emisor electromagnético TDR 1502B ${ }^{\circledR}$ (Time Domain Reflectrometry, Tektronic Inc., Beaverton, OR, USA) (Vera et al., 2009). Otras variables medidas fueron rendimiento de fruto total y calidad del fruto (ésta mediante clasificación por tamaño de fruto) en los calibres 3 (130 a $160 \mathrm{~g}), 4(160$ a $200 \mathrm{~g})$ y $5(>200$ g) todas estas categorías son consideradas como fruto comercial.

Los datos de rendimiento fueron procesados con análisis de varianza y la prueba de Tukey (5\%) para comparar los promedios de tratamientos; se relacionó también el agua evapotranspirada (ETr) con el rendimiento de fruto para obtener la eficiencia de uso de agua ( $\mathrm{kg}$ de fruto $\mathrm{m}^{-3}$ agua evapotranspirada). 


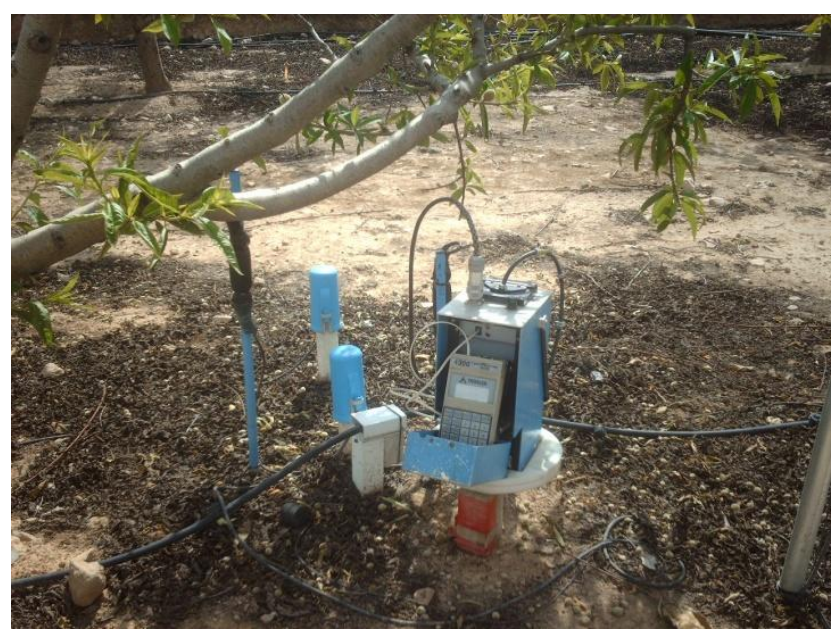

Figura 1. Sonda de neutrones utilizada para medir la humedad del punto en la rizosfera del melocotonero.

\section{RESULTADOS Y DISCUSIÓN}

Los diferentes tratamientos de riego tuvieron efecto en la evapotranspiración real (ETr) del árbol de melocotón. En la Figura 2 se muestra que a mayor cantidad de agua aplicada, también hay mayor consumo de agua por el árbol. T1 incrementó en $100 \%$ la cantidad de agua evapotranspirada $(1220 \mathrm{~mm})$ respecto al tratamiento inmediato inferior el T4 $(639 \mathrm{~mm})$, lo cual es importante en condiciones de escasez de agua como enfrenta cada año el mediterráneo español. Los otros tratamientos tuvieron aún menor ETr, con $476 \mathrm{~mm}$ para T2 y 495 para T3. Se observó que con un cambio en la frecuencia y cantidad de riego, el árbol se ajusta a estas condiciones y reduce o incrementa sus niveles de gasto evapotranspirativo (García et al., 2006). Las reducciones en el agua aplicada significan más de $5000 \mathrm{~m}^{3} \mathrm{ha}^{-1}$, lo cual es importante en condiciones de escasez del recurso. También se apreció que la ETo o evapotranspiración de referencia es muy similar a la ETr de T1.

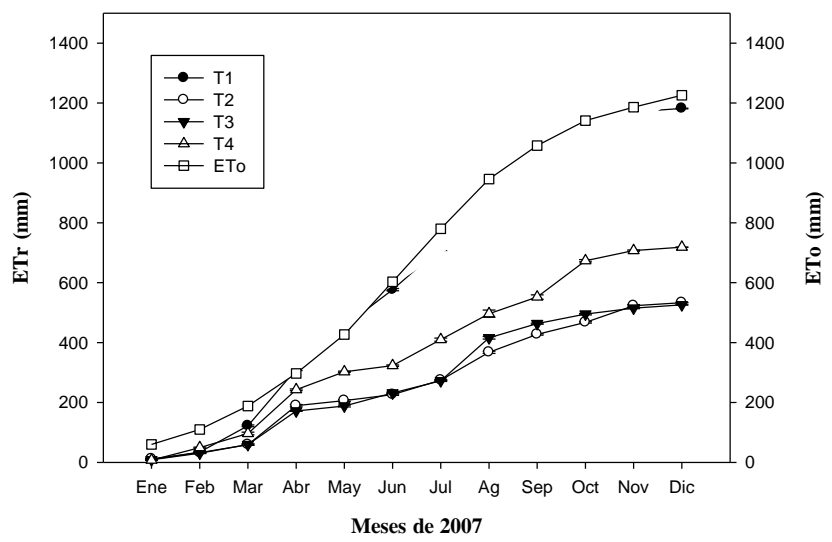

Figura 2. Evapotranspiración real (ETr) del melocotonero en cada tratamiento de manejo de agua y la evapotranspiración de referencia (ETo). $\mathrm{T} 1=$ riego a $120 \%$ de ETc; $\mathrm{T} 2=$ riego $50 \%$ de ETc; $\mathrm{T} 3=$ riego deficitario en postcosecha; $\mathrm{T} 4=$ riego basado en umbrales de humedad; ETo = evapotranspiración de referencia. Las barras acotadas son el error estándar de cada punto graficado.

El rendimiento de fruto comercial de $\mathrm{T} 1$ y $\mathrm{T} 4$ con 50.1 y $45.4 \mathrm{~kg} /$ árbol, respectivamente (Cuadro 1), superaron $(\mathrm{P} \leq 0.05)$ a los de T2 y T3 con 33.2 y $36.0 \mathrm{~kg} /$ árbol. Estos resultados indican claramente que a mayor cantidad de agua aplicada se tiene también un mayor rendimiento de fruto, con incrementos hasta de $18 \mathrm{~kg} /$ árbol, lo cual puede conducir a aceptar que más riego es mejor en la producción de melocotón. Sin embargo, el tratamiento T4 implica una reducción del riego, equivalente a más de 500 $\mathrm{mm}$ anuales sin reducción del rendimiento de fruto. En estudios anteriores, Girona et al. (2005) determinaron que reducción moderada del riego no afecta el rendimiento de fruto y aun cuando hay un leve efecto en el amarre éste no es determinante en el rendimiento; pero a diferencia de este trabajo, sólo redujeron hasta $35 \%$ la cantidad de agua aplicada, mientras que aquí se pudo reducir en más de $50 \%$ el riego aplicado.

Cuadro 1. Evapotranspiración real (ETr), rendimiento de fruto comercial y eficiencia en el uso de agua (EUA) en cuatro tratamientos de riego de melocotonero.

\begin{tabular}{lccccc}
\hline Tratamiento & $\begin{array}{c}\text { ETr } \\
(\mathrm{mm} / \mathrm{semana})\end{array}$ & $\begin{array}{c}\text { Drenaje } \\
(\mathrm{mm})\end{array}$ & $\begin{array}{c}\text { Riego } \\
(\mathrm{mm})\end{array}$ & $\begin{array}{c}\text { Rendimiento } \\
(\mathrm{kg} / \mathrm{a} r b o l)\end{array}$ & $\begin{array}{c}\text { EUA } \\
\left(\mathrm{kg} \mathrm{m}^{-3}\right)\end{array}$ \\
\hline T1 (Testigo) & $21.8 \mathrm{a}$ & 42.8 & 1221.1 & $50.1 \mathrm{a}$ & $2.08 \mathrm{~b}$ \\
T2 & $8.8 \mathrm{c}$ & 40.6 & 639.3 & $33.2 \mathrm{~b}$ & $2.77 \mathrm{ab}$ \\
T3 & $9.2 \mathrm{c}$ & 44.1 & 410.2 & $36.0 \mathrm{~b}$ & $3.05 \mathrm{a}$ \\
T4 & $12.2 \mathrm{~b}$ & 51.5 & 665.6 & $45.5 \mathrm{a}$ & $2.84 \mathrm{ab}$ \\
Lluvia (mm) & 275.2 & & & & 0.89 \\
DMS & 2.9 & & & 5.3 & \\
\hline
\end{tabular}

$\mathrm{T} 1$ = riego a $120 \%$ de ETc; $\mathrm{T} 2$ = riego $50 \%$ de ETc; T3 = riego deficitario en postcosecha; T4 = riego basado en umbrales de humedad. EUA $=\mathrm{kg}$ de fruto comercial por árbol y por $\mathrm{m}^{3}$ de agua evapotranspirada. Valores con la misma letra son iguales estadísticamente (Tukey, 0.05). DMS = diferencia mínima significativa. 
Con estos rendimientos totales por árbol, la eficiencia del uso del agua (EUA) fue mayor $(\mathrm{P} \leq 0.05)$ en $\mathrm{T} 3$ con $3.05 \mathrm{~kg}$ de fruto $\mathrm{m}^{-3}$ de agua evapotranspirada. No obstante, con los tratamientos $\mathrm{T} 4$ y $\mathrm{T} 2$ que implican menor aplicación de agua de riego, la EUA presenta similar significancia estadística que T3. El tratamiento con menor eficiencia de uso de agua fue el T1 con sólo $2.08 \mathrm{~kg} \mathrm{~m}^{-3}$, que evidencia la posibilidad de aplicar mejores prácticas de riego para aumentar el uso racional del agua (Cuadro 1).

En relación con la calidad de fruto producido (Cuadro $2)$, los tratamientos $\mathrm{T} 2$ y $\mathrm{T} 3$ redujeron $(\mathrm{P} \leq 0.05)$ la producción de fruto en los calibres mayores de $130 \mathrm{~g}$, al producir menos de $8 \mathrm{~kg} /$ árbol $^{1}$ en calibre 130 a $160 \mathrm{~g}$, menos de $2 \mathrm{~kg}$ en calibre 160 a $200 \mathrm{~g}$ y menos de $0.1 \mathrm{~kg}$ en calibres superiores ( $>200 \mathrm{~g})$. Estas cantidades son inferiores $(\mathrm{P} \leq 0.05)$ a las producidas por los tratamientos testigo y T4, que fueron superiores a $16.9 \mathrm{~kg}, 5.6 \mathrm{~kg}$ y $0.7 \mathrm{~kg} /$ arbol, para los citados calibres, respectivamente; cabe señalar que las producciones entre estos dos tratamientos fueron iguales en los tres calibres más grandes de fruto.

Sin embargo, la eficiencia en el uso del agua fue superior $(\mathrm{P} \leq 0.05)$ en $\mathrm{T} 4$ con $1.05 \mathrm{~kg} \mathrm{~m}^{-3}$ de agua evapotranspirada para el calibre $130 \mathrm{a} 160 \mathrm{~g}$, que el resto de los tratamientos, inclusive el testigo $\left(0.78 \mathrm{~kg} \mathrm{~m}^{-3} \mathrm{de}\right.$ agua evapotranspirada). En los calibres intermedios (160 a $200 \mathrm{~g}$ ), los tratamientos testigo T1 y T4 también fueron superiores $(\mathrm{P} \leq 0.05)$ que $\mathrm{T} 2$ y T3 en EUA, pero iguales entre sí ( $>0.3 \mathrm{~kg} \mathrm{~m}^{-3}$ de agua evapotranspirada).

Este análisis permite determinar que el ahorro de agua en melocotonero es posible sin menoscabo del rendimiento de fruto ni de su calidad, con el T4. El ahorro en EUA dado por los tratamientos T2 y T3 redujo tanto el rendimiento, como la calidad y la eficiencia de uso de agua. El tamaño final del fruto tal vez es influenciado por la disponibilidad de agua durante la fase inicial de crecimiento de fruto, como la argumentan Genard y Huguet (1996); esto sería válido para T2 mientras que para T3 la reducción se debe a la baja disponibilidad de agua en el año anterior, como argumentan Torrecillas et al. (2000). Nótese que con T4 el mantener un umbral de humedad estable no conduce a un déficit severo de agua y, por tanto, no afecta ni al rendimiento ni al tamaño final, pero sí hay un ahorro de riego y mayor eficiencia evapotranspirativa.

La cantidad total de fruto comercial (Cuadro 3) no fue disminuida por la reducción de $>500 \mathrm{~mm}$ de riego que hubo en $\mathrm{T} 4$, que con $23.3 \mathrm{~kg} /$ árbol produjo la misma cantidad de fruto que el testigo con $27.4 \mathrm{~kg} /$ árbol, con porcentajes superiores a $50 \%$ de fruto comercializable. Una mayor reducción del riego afectaría la producción del fruto comercial, ya que tanto T2 como T3 con menos de $11 \mathrm{~kg} /$ árbol redujeron significativamente el rendimiento y sus porcentajes de fruto comercial fueron menores a $30 \%$ de su rendimiento total; el T2 puede llegar a producir casi $90 \%$ de su fruto en calidad no comercial, lo cual afectaría definitivamente la rentabilidad del cultivo, porque la reducción del riego es a niveles drásticos. Es interesante notar que el fruto de desecho en los tratamientos T1 (testigo) y T4 son iguales entre sí (aprox. $22.2 \mathrm{~kg} /$ árbol) y al resto de tratamientos.

Cuadro 3. Fruto comercial y fruto de desecho en cuatro tratamientos de riego de melocotón en el Mediterráneo español.

\begin{tabular}{lccccc}
\hline Tratamiento & \multicolumn{2}{c}{ Fruto comercial } & & \multicolumn{2}{c}{ Fruto de desecho } \\
\cline { 2 - 3 } \cline { 2 - 3 } & $(>130 \mathrm{~g})$ & $(\%)$ & & $(<130 \mathrm{~g})$ & $(\%)$ \\
\hline T1 (Testigo) & $27.4 \mathrm{a}$ & 54.8 & & $22.6 \mathrm{a}$ & 45.2 \\
T2 & $3.9 \mathrm{c}$ & 11.7 & & $29.3 \mathrm{a}$ & 88.2 \\
T3 & $10.8 \mathrm{~b}$ & 29.9 & & $25.2 \mathrm{a}$ & 70.1 \\
T4 & $23.3 \mathrm{a}$ & 51.2 & & $22.2 \mathrm{a}$ & 48.8 \\
DMS & 6.3 & & & 7.4 &
\end{tabular}

$\mathrm{T} 1=$ riego a $120 \%$ de $\mathrm{ETc} ; \mathrm{T} 2=$ riego $50 \%$ de $\mathrm{ETc} ; \mathrm{T} 3=$ riego deficitario en postcosecha; $\mathrm{T} 4$ = riego basado en umbrales de humedad. Valores con la misma letra son iguales estadísticamente (Tukey, 0.05). DMS = diferencia mínima significativa.

Cuadro 2. Promedios de rendimiento comercial de fruto de melocotón (kg/árbol) por calibre ( $>130 \mathrm{~g})$ y eficiencia en el uso de agua ( $\mathrm{kg}$ de fruto por $\mathrm{m}^{3}$ de agua evapotranspirada).

\begin{tabular}{lcccccc}
\hline & \multicolumn{7}{c}{ Calibre de fruto } \\
\cline { 2 - 7 } Trat. & $130 \mathrm{a} 160 \mathrm{~g}$ & EUA & $160 \mathrm{a} \mathrm{200 \textrm {g }}$ & EUA & $>200 \mathrm{~g}$ & EUA \\
\hline T1 (Testigo) & $18.8 \mathrm{a}$ & $0.78 \mathrm{ab}$ & $7.41 \mathrm{a}$ & $0.31 \mathrm{ab}$ & $1.28 \mathrm{a}$ & $0.05 \mathrm{a}$ \\
T2 & $3.2 \mathrm{~b}$ & $0.27 \mathrm{c}$ & $0.61 \mathrm{~b}$ & $0.05 \mathrm{~b}$ & $0.06 \mathrm{~b}$ & $0.01 \mathrm{~b}$ \\
T3 & $8.2 \mathrm{~b}$ & $0.70 \mathrm{~b}$ & $2.33 \mathrm{~b}$ & $0.20 \mathrm{ab}$ & $0.21 \mathrm{~b}$ & $0.02 \mathrm{ab}$ \\
T4 & $16.9 \mathrm{a}$ & $1.05 \mathrm{a}$ & $5.66 \mathrm{a}$ & $0.35 \mathrm{a}$ & $0.75 \mathrm{ab}$ & $0.05 \mathrm{a}$ \\
DMS & 4.5 & 0.31 & 2.5 & 0.25 & 0.71 & 0.03 \\
\hline
\end{tabular}

$\mathrm{T} 1=$ riego a $120 \%$ de ETc; $\mathrm{T} 2=$ riego $50 \%$ de ETc; T3 = riego deficitario en postcosecha; $\mathrm{T} 4$ = riego basado en umbrales de humedad. EUA = eficiencia de uso de agua ( $\mathrm{kg}$ de fruto comercial por árbol y por $\mathrm{m}^{-3}$ de agua evapotranspirada). Valores con la misma letra son iguales estadísticamente (Tukey, 0.05). DMS $=$ diferencia mínima significativa. 


\section{CONCLUSIONES}

La evapotranspiración del cultivo del melocotón variedad 'Flordastar' en el mediterráneo español se incrementó de manera directamente proporcional al riego suministrado al cultivo, mientras que la eficiencia de uso de agua fue mayor en los tratamientos con menor lámina de riego. El rendimiento total de fruto y el rendimiento de calidad comercial (fruto $>120 \mathrm{~g}$ ) fueron más altos en los tratamientos testigo y el basado en umbrales de humedad, pero este último con reducción de riego de $54 \%$. Si se reduce en mayor grado, el riego del melocotón, se reduce también el rendimiento y la calidad de fruto. La eficiencia de uso de agua se puede mantener en un nivel alto con alto rendimiento y calidad de fruto, mediante el tratamiento basado en umbrales de humedad (T4).

\section{AGRADECIMIENTOS}

A la Fundación Séneca (Murcia, Proy: 03130/PI/05) y el CONACYT (Reg 74696), por el financiamiento otorgado para esta investigación.

\section{BIBLIOGRAFÍA}

Abrisqueta J M, O Mounzer, S Álvarez, W Conejero, Y García, L M Tapia, J Vera, I Abrisqueta, M C Ruiz (2008) Root dynamics of peach trees submitted to partial rootzone drying and continuous deficit irrigation. Agric. Water Manage. 95:959-967

Allen R G, R S Pereira, D Raes, M Smith (1998) Crop Evapotranspiration. Guidelines for Computing Crop Water Requierements. FAO. Irrigation and Drainage. Paper 56, Roma, Italia. $120 \mathrm{p}$.

Arregui A M, M Quemada (2006) Drainage and nitrate leaching in a crop rotation under different $\mathrm{N}$-fertilizer strategies: application of capacitance probes. Plant and Soil 288:57-69.
Ballesteros R R (2005) Agua, la botella medio lleno. La Economía 77:1-3.

Conejero W, J J Alarcón, Y García-Orellana, E Nicolás, A Torrecillas (2007) Evaluation of sap flow and trunk diameter sensors used for irrigation scheduling in early maturing peach trees. Tree Physiol. 27:1753-1759.

García B J, L Rincón, J Sáez (2006) The response of 'Catherine' cultivar peach trees grafted on sixteen rootstocks under four irrigation levels. Acta Hort. 658:23.

Genard M, J G Huguet (1996) Modeling the response of peach fruit growth to water stress. Tree Physiol. 16:407-415.

Girona J, M Gelly, M Mata, A Arbonès, J Rufat, J Marsal (2005) Peach tree response to single and combined deficit irrigation regimes in deep soils. Agric. Water Manage. 72:97-108.

Martinez C A, P Romero, J I Fernández (2007) Técnicas de riego deficitario en el cultivo de la vid. Vida Rural 444:17-21

Mounzer O H, J Vera, L M Tapia, Y. García, W Conejero, I Abrisqueta, M C Ruiz, J M Abrisqueta (2008a) Irrigation scheduling of peach trees by continuous measurement of soil water status. Agrociencia 42:857-868.

Mounzer O H, R Mendoza, I Abrisqueta, L M Tapia, J M Abrisqueta, J Vera, M C Ruiz (2008b). Soil water content measured by FDR probes and thresholds for drip irrigation management in peach trees. Agric. Téc. Méx. 34:313-322.

Ortuño M F, Y García, W Conejero, M C Ruiz, J J Alarcón, A Torrecillas (2006) Stem and leaf water potentials, gas exchange, sap flow and trunk diameter fluctuations for detecting water stress in lemon trees. Trees Struct. Funct. 20:1-8.

Playan E, L Mateos (2006) Modernization and optimization of irrigation systems to increase water productivity. Agric. Water Manage. 80:100-116.

Soil Survey Staff (1998) Keys to Soil Taxonomy. 10th ed. USDANatural Resources Conservation Service, Washington, D.C. $341 \mathrm{p}$.

Torrecillas A, R Domingo, R Galego, M C Ruiz-Sánchez (2000) Apricot tree response to irrigation withholding at different phenological periods. Sci. Hort. 85:201-215.

Vera J, O Mounzer, M C Ruiz, I Abrisqueta, L M Tapia, J M Abrisqueta (2009) Soil water balance trial involving capacitance and neutron probe measurements. Agric. Water Manage. 96:905-911. 